\title{
Impact of medication review, within a shared decision-making framework, on deprescribing in people living in care homes
}

\author{
Wasim Baqir, ${ }^{1,2}$ Julian Hughes, ${ }^{1}$ Tania Jones, ${ }^{3}$ Steven Barrett, ${ }^{1}$ Nisha Desai, ${ }^{1}$ \\ Richard Copeland, ${ }^{1}$ David Campbell, ${ }^{1}$ Annie Laverty ${ }^{1}$
}

\begin{abstract}
Department of Pharmacy, Northumbria Healthcare NHS Foundation Trust, North Shields, UK

${ }^{2}$ Department of Pharmacy, University of Bradford, School of Pharmacy, Bradford, Bradford, UK

${ }^{3}$ Department of Pharmacy Health and Well-being, University of Sunderland, Sunderland, UK
\end{abstract}

\section{Correspondence to} Dr Wasim Baqir, Department of Pharmacy, North Tyneside Hospital, Rake Lane, North Shields NE29 8NH, UK: Wasim.baqir@nhs.net

Received 25 January 2016 Revised 31 March 2016 Accepted 11 April 2016

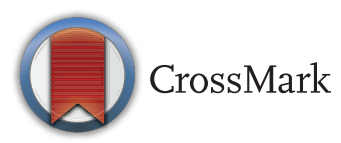

To cite: Baqir W, Hughes J, Jones T, et al. Eur J Hosp Pharm 2017:24:30-33.

\begin{abstract}
Objectives The key objectives of this study were to quantify extent of prescribing, reasons for deprescribing, common therapeutic groups of medicines deprescribed and adverse events.
\end{abstract}

Methods A retrospective analysis was carried out on a quality improvement project where 422 care home residents in 20 care homes received a medicines optimisation review with a pharmacist and other members of the healthcare team (general medical practitioner, care home nurse). Data on number, type and cost of medicines were collected. Statistical analysis was performed to test for differences between pharmacist-only review and the pharmacist plus general practitioner (GP), and to identify any correlation between the original number of medicines and the number of medicines stopped.

Results Of the 422 patients reviewed, 298 (70.6\%) had at least one medicine stopped with 704 medicines being stopped. This represented $19.5 \%$ of the medicines originally prescribed (3602 medicines). There was no statistically significant difference between pharmacist only and pharmacist plus GP in terms of stopping medicines. The main groups of medicines stopped were laxatives, skin products and bone protection. There was weak correlation between the original number of medicines prescribed and the number stopped. Conclusions This study has shown that medicines optimisation reviews can lead to a reduction in polypharmacy for care home residents through a deprescribing process. Patients' medicine regimens were simplified and optimised while making financial significant savings for the National Health Service.

\section{INTRODUCTION}

While polypharmacy can be essential to improve the quality of life for many patients, it can also lead to increased risks of adverse drug reactions, drug interactions and reduced medicines adherence. There is a body of evidence to suggest that potentially inappropriate prescribing and polypharmacy is common in elderly populations residing in nursing home settings. ${ }^{1}{ }^{2}$ Targeting and reducing such prescribing should be a key priority for clinicians, solely to reduce negative clinical outcomes and the adverse effects of inappropriate drugs and to reduce the financial burden on healthcare providers.

To minimise these effects, a key component of medicines optimisation is the avoidance of unnecessary or harmful medicines, with studies showing that input from a multidisciplinary team (MDT) or a pharmacist-led review can facilitate the reduction of polypharmacy. ${ }^{3-5}$

Deprescribing is a term frequently used to describe the planned reduction and stopping of medication. ${ }^{6-8}$ It is an important part of the prescribing process as medicines should be regularly reviewed and when a medicine stops benefiting a patient it should be stopped.

Guidance for withdrawing medication, as with starting treatments, will help to ensure that deprescribing becomes part of a prescribing process. However, there is little support for practitioners who wish to stop medicines. Solutions to support deprescribing include tools which identify potentially inappropriate medication, such as the STOPP-START tool ${ }^{9}$ and Beers criteria. ${ }^{10}$ Although studies have demonstrated their impact on reducing inappropriate medicines and improving safety, they do not cover all medicines and many patients are prescribed medicines that no longer necessary. All patients should receive a regular benefit-harm assessment of their medication as circumstances change, bearing in mind age and comorbidities, to identify medicines which are providing little benefit. Such tools lack outcome measures such as overall health or medication-related adverse events. ${ }^{11} 12$ The criteria used to stop medicines which have brought no therapeutic benefit are usually a matter of clinical judgement. An alternative approach detailed by Scott et $a l^{13}$ suggests a stepwise approach where the clinician should, for each medicine, assess reasons for treatment, consider risk, consider future benefits, prioritise those medicines with the lowest chance of harm from withdrawal and monitor.

Rational medication use should involve patients in decision making about their medication and consider when medication may no longer be beneficial. Prescribers must acknowledge and overcome uncertainties inherent in deprescribing of medication. ${ }^{14}$ Barriers to patient-centred deprescribing include disagreement with the appropriateness of cessation, fear of consequences of cessation, absence of a process for cessation and negative influences on patients' decisions to cease medication. ${ }^{15}$

There are emerging studies on the potential benefits of stopping medicines in older people with multiple medicines and medical conditions. ${ }^{16}$ Todd et al and Lindsay et al showed the potential to stop medicines in patients with lung cancer. ${ }^{17}{ }^{18}$ Todd et al showed that the majority of their patients with lung cancer were taking medicines that could be 
stopped and in some cases were taking medicines that interacted with the cancer treatment. ${ }^{18}$

This study aimed to investigate whether medicines review led by pharmacists would lead to deprescribing of medications. Secondary aims were to assess the reasons for and impact of deprescribing. The key objectives of this study were to quantify extent of prescribing, reasons for deprescribing, common therapeutic groups of medicines deprescribed and adverse events.

\section{METHODS}

This was a retrospective analysis of the Shine Medication Optimisation Project, a quality improvement (QI) project where care home residents were reviewed and medicines optimised within a shared decision-making framework. ${ }^{19}$ A key area that the clinical team were keen to explore was the deprescribing of medicines that had no clinical benefit as well as those highlighted by tools such as STOPP. A patient-centred approach was used, where the answer to three questions (Northumbria 3Q) were discussed with residents in 20 care homes and joint decisions made. ${ }^{19}$ The three questions were: (i) Is there an indication for the medication (ie, where the medicine is neither treating nor preventing any disease nor alleviating symptoms)? (ii) Is the indication appropriate when comorbidity and current clinical situation is taken into consideration (eg, bone protection treatments for bed-bound residents)? and (iii) Is the medication safe? ${ }^{19}$ Clinical judgement backed up by a review of the clinical notes was used to assess appropriateness of medicine. The final decision to stop or continue the medicines was made jointly with the patient or their advocate.

All care homes across a Clinical Commissioning Group (CCG) were invited to participate, with residents based in 20 homes receiving medication optimisation reviews. As this was a QI project, care homes were not randomly selected, but selected based on their willingness and on their general medical practice to participate in the project. Care homes across two CCGs in North East England were invited to participate. Of the 20 care homes, 15 were mixed (nursing, elderly and mentally infirm and residential) with three being residential and two nursing only.

The review process involved a notes based, pharmacist-led review of medicines, where the Northumbria 3Q approach was applied to each medicine, that is, was there an indication, was the indication appropriate and was it safe?. Additionally, medicines missing that could be beneficial (eg, START medicines) were identified. This was followed by a MDT meeting involving pharmacists and care home nurses, with other professionals (eg, general medical practitioners and mental health professionals) joining when required. At the MDT, the information from the pharmacist-led review was discussed and an action plan was formulated (eg, stop, start or change medicines). Whenever possible, the final decisions were made with patients and their families, once the risks and benefits of the proposed intervention were explained. After the review, the project database was updated to show medicines taken before review, medicines stopped, started or changed and any other interventions made.

In total, 422 residents were reviewed, with pharmacists and other professionals making 1346 interventions jointly with care home residents. ${ }^{19}$ The most common intervention was to stop medicines, accounting for 704 medicines stopped in 298 $(70.6 \%)$ of patients. ${ }^{19}$

The project database was analysed and data presented descriptively. The number and type of medicines stopped as well as the cost saving associated with deprescribing was reported. The reasons why medicines were stopped were collated and these data were extracted from the project database for this study. All medicines prices were calculated using the National Health Service (NHS) Drug Tariff and savings annualised, thus making the assumption that the medicine would have been taken for another 12 months after deprescribing. The Kings Fund predicts a $65 \%$ increase in patients needing advanced care by $2030 .^{20}$ The Nuffield Trust have estimated a median length of stay of 18 months for people admitted to permanent residential care. ${ }^{21}$ This is consistent with a BUPA 2012 survey of its care homes which demonstrated a median length of stay of 20 months. ${ }^{22}$

In the project there were two models of working: pharmacists working independently of general practitioners (GPs), making prescribing decisions, including decisions to stop medicines, and prescribing decisions made jointly by pharmacists and GPs. Both models of working were part of a shared decision-making approach with residents or their families.

All patients were followed up 1 month after the intervention to ensure that any changes made had no adverse events and if so, those events were managed. All adverse events were entered into the database. Care home nurses were able to contact the pharmacist if they had any concerns.

IBM SPSS Statistics (V.21) was used to determine statistical differences, on the basis of a test, between number of medicines stopped, between pharmacists working independently or with general medical practitioners. To understand whether the deprescribing was affected by the original number of medicines prescribed to the patient, a Pearson' correlation was performed.

This project was funded by the Health Foundation as part of the Shine 2012 programme. This was run as a QI project. Advice on ethical approval was sought from the Trust's research and development lead. It was agreed that ethical approval for the QI project and this subsequent analysis were not required.

\section{RESULTS}

Four hundred and twenty-two residents were reviewed in 20 care homes ( 2 residential, 3 nursing and 15 mixed) between January 2013 and March 2014. The average age of the cohort was 85.5 years with $77.7 \%$ being female. The 422 residents were collectively taking 3602 medicines before the review, with $704(19.5 \%)$ stopped in 298 residents during the review. The 298 residents who had medicines stopped were taking a total of 1346 medicines before review, with 704 (52.3\%) of their medicines stopped. The mean number of medicines stopped was 2.36 (SD 1.53) ranging from 0 to 9 . There was no statistical difference between numbers of medicine stopped by pharmacist prescribers (53.4\% stopped) and numbers stopped $(51.9 \%)$ where GPs were involved ( $\mathrm{p}=0.9702 ; 95 \% \mathrm{CI}-0.39$ to 0.38 ).

Of the 704 medicines that were stopped, 142 were acute medicines and 562 were regular repeat medicines. The primary reason for stopping medicines was that the medicine had no current indications $(\mathrm{n}=400 ; 56.8 \%)$ with $15.9 \%, 8.7 \%$ and $6.5 \%$ of deprescribing accounted for by, respectively, patient choice, the indication no longer being appropriate and safety reasons (table 1 ).

Medicines were stopped from all but three (malignant disease, immunology and anaesthesia) sections of the British National Formulary. The most common groups of medicines deprescribed were laxatives $(14.5 \%)$, skin products $(8.4 \%)$, bone protection drugs (7\%), acid-regulating medicines (5.4\%), antidepressants (4.7\%), antihypertensives $(4.3 \%)$ and lipid-regulating medicines (4.3\%) (table 2). All medicines were stopped after involving residents and/or their families/advocates and taking their views into account. $^{19}$ 
Many residents were prescribed a number of laxatives and the main drugs stopped were sodium docusate, macrogols and senna. Bone protection medicines were all stopped because the resident was immobile (bed or chair bound) and at low risk of falls. Many acid-regulating drugs were no longer indicated (eg, they were originally prescribed short term). Antidepressants were deprescribed after consultation with resident, family and care home staff, sometimes also involving the relevant old age psychiatrist. Hypertension medicines were only stopped where blood pressure was low or where there was a falls risk. Lipid-regulating medicines were mainly statins and were stopped after discussing their benefits and risks.

All residents were monitored following any deprescribing and adverse events documented, with every patient being followed up 1 month after the intervention. Only seven events $(0.99 \%)$ were reported (table 3 ). The events were all minor and were reversed. In one case, the patient became very weepy and tearful and the antidepressant (citalopram) was restarted. For four situations another medicine was started, and the for the remaining two cases the medicine was started at a lower dose and monitoring was put into place.

The relationship between the number of medicines originally prescribed and number stopped was investigated using Pearson's correlation. There was a weak positive relationship $(r=0.333)$ which tailed off at 15 medicines originally prescribed.

The assumption that each medicines would have been taken for another year was made when measuring the financial impact of deprescribing. The medicines stopped amounted to $£ 65471$ in terms of annualised savings. ${ }^{19}$

\section{DISCUSSION}

This study aimed to uncover the extent to which deprescribing could be conducted using a structured medication review within a MDT across 20 nursing homes in North East England. Overall, 1 in 20 medicines were stopped for every resident reviewed, with medicines being stopped in over two thirds of residents reviewed. With this ageing cohort, it is expected that there will be multiple comorbidities and complex clinical presentations. It is, perhaps, not surprising that patients were prescribed an average of nine medicines per resident before the medication reviews, yet this was reduced to an average of seven medicines per person reviewed.

Using two models of delivery for the service (pharmacist actioning decisions or pharmacist and GP actioning decisions), there was no statistical difference in number of medicines deprescribed between the two methods. This has implications for the current NHS where general medical practitioner resource and workload have been focus of much media attention. A model where one practitioner (the pharmacist) can have a similar

Table 1 Reasons why medicines were deprescribed

\begin{tabular}{lr}
\hline Reason & Medicines ( $)$ \\
\hline No indication & 400 \\
Patient refusing medicine & 112 \\
Indication not appropriate & 61 \\
Safety & 46 \\
Ineffective treatment & 29 \\
Therapeutic Switch & 17 \\
Duplication & 12 \\
Other & 27 \\
\hline
\end{tabular}

Table 2 Common groups of medicines deprescribed

\begin{tabular}{lrc}
\hline Type of drug & Number & Per cent \\
\hline Laxatives & 102 & 14.4 \\
Skin & 59 & 8.4 \\
Bone protection & 49 & 7.0 \\
Acid regulating & 38 & 5.4 \\
Antidepressants & 33 & 4.7 \\
Hypertension & 30 & 4.3 \\
Lipid regulation & 30 & 4.3 \\
Others & 363 & 51.5 \\
\hline
\end{tabular}

impact to both a pharmacist and GP will be more desirable in terms of resource and cost-effectiveness.

The fact that there is some correlation between number of medicines prescribed and number stopped could mean that patients on higher number of medicines can be targeted for reviews. However, further work will be needed to establish if this will result in better outcomes for patients (eg, avoiding hospital admissions).

The main reason for stopping a medicine was when there was no current indication for the drug, followed by discontinuation on patient request. Other studies have found reasons for polypharmacy include the prescribing of duplicate medicines or the use of medicines no longer required which have yet to be removed from the repeat prescription. ${ }^{23} 24$

It was determined that there was only a weak positive relationship between the number of drugs suitable for discontinuation and the number of medicines taken before review. It can be concluded that it is not always necessary for patients to be taking large numbers of drugs to allow successful deprescribing to take place.

There were nine adverse effects reported thought to be related to the cessation of a medicine during the study. The events were discussed with GP colleagues and not deemed serious and they were identified and appropriately rectified by either monitoring the patient or reinstating the drug or an alternative. Through careful review using a multidisciplinary approach, deprescribing can be performed effectively and safely. Garfinkel cited fear of adverse events following deprescribing as a barrier so the results of this are reassuring. ${ }^{11}$ It is also clear that appropriate monitoring postcessation is important to pick up any untoward effects that may occur. One of the limitations in attributing adverse effects to the cessation of a drug is that is it difficult to be certain of causation, bearing in mind the complexity of comorbidities in the older population.

Table 3 Adverse events caused by deprescribing

\begin{tabular}{|c|c|c|}
\hline Event & Cause & Action \\
\hline $\begin{array}{l}\text { Hypertensive (BP } \\
\text { increased from } 130 / 80 \\
\text { to } 158 / 80 \text { ) }\end{array}$ & $\begin{array}{l}\text { Stopped } \\
\text { bendroflumethiazide }\end{array}$ & $\begin{array}{l}\text { Monitor but leave off } \\
\text { bendroflumethiazide }\end{array}$ \\
\hline Swelling legs & Furosemide stopped & Restarted but lower dose \\
\hline UTI & Stopped trimethoprim & $\begin{array}{l}\text { Treated and restarted } \\
\text { prophylaxis }\end{array}$ \\
\hline BP $170 / 90$ & $\begin{array}{l}\text { Amlodipine stopped } \\
\text { (leg swelling) }\end{array}$ & Start another hypertensive \\
\hline Weepy and tearful & Stopped citalopram & Restarted \\
\hline Blood glucose raised & Stopped metformin & Increase insulin \\
\hline GI-heartburn & Stopped omeprazole & Start lansoprazole \\
\hline
\end{tabular}

Baqir W, et al. Eur J Hosp Pharm 2017;24:30-33. doi:10.1136/ejhpharm-2016-000900 
To assess the financial impact, we assumed that residents would have taken medicines for at least another 12 months after it was deprescribed. This is an underestimate as on average care home residents live for 18 months. ${ }^{21}$ It is a limitation of this study that actual lifespan was not measured. Significant savings were shown from the primary care medicines budget (in excess of $£ 60000$ ), which if scaled up could potentially reduce healthcare expenditure globally. There is also the possibility that sequelae from inappropriate prescribing may have increased healthcare costs further, had the drug not been stopped. It is impossible to predict the extent to which deprescribing may have prevented such complications of therapy. The Shine project reported an average reduction in nurse time administering medicines of $6.6 \mathrm{~h}$ per home per week ${ }^{19}$ and although not formally evaluated in this study, nurses stated in feedback that this was a result of less medicines being administered to their residents.

A further limitation of this study is that the impact of deprescribing on pharmaceutical waste was not measured. Further research into financial savings from reduced medicines waste would be useful. The longer term clinical outcomes for the patients undergoing deprescribing also remain uncertain. In the short term, patients tolerated the reduction in medication reasonably well, but data collection was not continued over an extended period which would have allowed any longer term complications to be detected.

Another limitation of the study was the measure of intervention quality. Pharmacist and physician interventions were not externally validated by an independent clinician for standardisation. It can be assumed that approaches to deprescribing will depend on the individual clinician, based on their experience and profession.

Deprescribing medicines lacks the robust evidence that evidence-based medicine offers prescribers when starting medicines. Garfinkel $e a l^{11}$ argue that much of the evidence of medicines is inappropriate for older and especially frail older people. and the numerous tools available to tackle excess and inappropriate prescribing there exists. Much like the approach take in this study, Scott $e t a l^{13}$ proposes a holistic review, questioning each medicine.

In conclusion, this study has shown that structured reviews with patient involvement and a multidisciplinary approach can lead to a reduction in polypharmacy for care home residents through a deprescribing process. Patients' medicine regimens were simplified and optimised while making financially significant savings for the NHS.

Key messages

What is already known on this subject

- Polypharmacy can lead to adverse events.

- Deprescribing medicines can improve quality and reduce healthcare costs.

- Deprescribing is difficult with few tools, guidelines and little evidence to support practitioners.

\section{What this study adds}

- Structured medicines optimisation reviews allow medicines to be deprescribed safely in a care home setting.

- Most medicines stopped had no indication or purpose.

- Medicines can be stopped across a wide range of therapeutic areas.
Twitter Follow Wasim Baqir at @wazzedagain

Contributors WB, JH, SB, ND, AL, RC, DC conceptualised the Shine project with $W B, J H, S B$ and ND running the project. This retrospective analysis was undertaken by WB and TJ. All authors contributed to the original manuscript. WB, JH and TJ updated the resubmitted manuscript.

Funding This project was funded by the Health Foundation SHINE 2012 Programme.

Competing interests None declared.

Provenance and peer review Not commissioned; externally peer reviewed.

Data sharing statement This is a retrospective analysis of the deprescribing component of the Shine care home project, All data have been summarised and presented, including data on adverse events resulting from deprescribing. The team is willing to share all data from the project. Please contact lead author (WB).

\section{REFERENCES}

1 Alldred DP, Raynor DK, Hughes C, et al. Interventions to optimise prescribing for older people in care homes. Cochrane Database Syst Rev 2013;2:CD009095.

2 Ruths S. Straand J, Nygaard HA. Multidisciplinary medication review in nursing home residents: what are the most significant drug-related problems? The Bergen District Nursing Home (BEDNURS) study. Qual Saf Health Care 2003;12:176-80.

3 Crotty M, Halbert J, Rowett $D$, et al. An outreach geriatric medication advisory service in residential aged care: a randomised controlled trial of case conferencing. Age Ageing 2004;33:612-17.

4 Zermansky AG, Alldred DP, Petty DR, et al. Clinical medication review by a pharmacist of elderly people living in care homes-randomised controlled trial. Age Ageing 2006:35:586-91.

5 Furniss L, Burns A, Craig SK, et al. Effects of a pharmacist's medication review in nursing homes. Randomised controlled trial. Br J Psychiatry 2000;176:563-7.

6 [No authors listed]. Describing deprescribing. Drug Ther Bull 2014;52:25

7 Alldred DP. Deprescribing: a brave new world? Int J Pharm Prac 2014;22:2-3.

8 Frank C. Deprescribing: a new word to guide medication review. CMAJ 2014;186:407-8

9 Gallagher P, O'Mahony D. STOPP (Screening Tool of Older Persons' potentially inappropriate Prescriptions): application to acutely ill elderly patients and comparison with Beers' criteria. Age Ageing 2008;37:673-9.

10 American Geriatrics Society Beers Criteria Update Expert Panel. Updated beers criteria for potentially inappropriate medication use in older adults. J Am Geriatr Soc 2012;60:616-31

11 Garfinkel, D., Ithan B, Bahat G. Routine deprescribing of chronic medications to combat polypharmacy. Ther Adv Drug Saf 2015;6:212-33.

12 Gnjidic, D., Le Couteur DG, Kouladjian L, et al. Deprescribing trials: methods to reduce polypharmacy and the impact on prescribing and clinical outcomes. Clin Geriatr Med 2012:28:237-53.

13 Scott IA., Hilmer SN, Reeve E, et al. Reducing inappropriate polypharmacy: the process of deprescribing. JAMA Intern Med 2015;175:827-34.

14 Todd A, Holmes HM. Recommendations to support deprescribing medications late in life. Int J Clin Pharm 2015;37:678-81.

15 Reeve $\mathrm{E}$, To J, Hendrix I, et al. Patient barriers to and enablers of deprescribing: a systematic review. Drugs Aging 2013;30:793-807.

16 Gnjidic, D., Le Couteur DG, Hilmer SN. Discontinuing drug treatments. BMJ 2014;349:g7013

17 Lindsay J, Dooley M, Martin J, et al. Reducing potentially inappropriate medications in palliative cancer patients: evidence to support deprescribing approaches. Support Care Cancer 2014;22:1113-19.

18 Todd A, Williamson S, Husband A, et al. Patients with advanced lung cancer: is there scope to discontinue inappropriate medication? Int J Clin Pharm 2013;35:181-4.

19 Baqir W, Barrett S, Desai N, et al. A clinico-ethical framework for multidisciplinary review of medication in nursing homes. BMJ Qual Improv Rep 2014;3:u203261. w2538-u.w2538.

20 The Kings Fund. Care demands and dementia. http://www.kingsfund.org.uk/ time-to-think-differently/trends/disease-and-disability/care-demands-dementia (accessed 25 Jan 2016).

21 Stevenson A, Roberts A. Estimating length of stay in publicly-funded residential and nursing care homes: a retrospective analysis using linked administrative data sets. BMC Health Services Research 2012:12:377.

22 BUPA. A profile of residents in BUPA care homes: results from the 2012 BUPA Census. http://www.cpa.org.uk/information/reviews/Bupa-Census-2012.pdf (accessed 25 Jan 2016).

23 Finkers F, Maring JG, Boersma $F$, et al. A study of medication reviews to identify drug-related problems of polypharmacy patients in the Dutch nursing home setting. J Clin Pharm Ther 2007;32:469-76.

24 Somers $M$, Rose $E$, Simmonds $D$, et al. Quality use of medicines in residential aged care. Aust Fam Physician 2010;39:413-16. 